\section{OP0082 FIBROSIS AND MICROANGIOPATHY ARE THE MAIN HISTOPATHOLOGICAL HALLMARKS OF SCLERODERMA-RELATED MYOPATHY}

C. Corallo ${ }^{1}$, M. Cutolo $^{2}$, S. Soldano ${ }^{2}$, N. Volpi ${ }^{1}$, D. Franci ${ }^{1}$, A. Montella ${ }^{1}$, C. Chirico ${ }^{1}$, R. Nuti ${ }^{1}$, N. Giordano ${ }^{1} .{ }^{1}$ Medicine, Surgery and Neurosciences, University of Siena, Siena; ${ }^{2}$ Research Laboratory and Academic Division of Clinical Rheumatology, Department of Internal Medicine, University of Genoa, Genoa, Italy

Background: Systemic sclerosis (scleroderma, SSc) is an autoimmune connective tissue disease characterized by skin and internal organ fibrosis, coupled with widespread vascular pathology. Skeletal muscle involvement in SSc has often been considered as a minor component of the disease associated with disuse.

Objectives: The goal of this study is to identify specific histopathological hallmarks of skeletal muscle involvement in SSc.

Methods: A total of $50 \mathrm{SSc}$ patients presenting clinical, serological and electromyographic (EMG) features of muscle weakness, were enrolled. Patients underwent vastus lateralis biopsy, assessed for individual pathologic features including fibrosis [type I collagen (Coll-I), transforming growth factor $\beta$ (TGF- $\beta$ )], microangiopathy [cluster of differentiation 31 (CD31), pro-angiogenic vascular endothelial growth factor A (VEGF-A), anti-angiogenic VEGF-A165b], immune/ inflammatory response [CD4, CD8, CD20, human leucocyte antigens ABC (HLA$A B C)]$, and membranolytic attack complex (MAC). SSc biopsies were compared to biopsies of $(n=50)$ idiopathic inflammatory myopathies (IIMs) and to $(n$ $=50$ ) noninflammatory myopathies (NIMs). Ultrastructural abnormalities of SSc myopathy were also analyzed by transmission electron microscopy (TEM).

Results: Fibrosis in SSc myopathy $(90 \%)$ is higher compared to IIM $(30 \%$, $p<0.05)$ and to NIM $(15 \%, p<0.05)$. Vascular involvement is dominant in SSc muscle $(95 \%)$, and in IIM $(75 \%)$ compared to NIM $(20 \%, p<0.05)$. In particular, CD31 shows loss of endomysial vessels in SSc myopathy with respect to IIM $(p<0.05)$ and to NIM $(p<0.01)$. VEGF-A is downregulated in SSc myopathy compared to IIM $(p<0.05)$ and to NIM $(p<0.05)$, while VEGFA165b is upregulated in SSc myopathy. The SSc immune/inflammatory response suggested humoral process with majority $(80 \%)$ HLA-ABC fibral neoexpression and complement deposits on endomysial capillaries MAC, compared to IIM ( $p$ $<0.05)$, characterized by CD4+/CD8+/B-cell infiltrate, and to NIM $(p<0.05)$. TEM analysis showed SSc vascular alterations consisting of thickening and lamination of basement membrane and endothelial cell 'swelling' coupled to endomysial/perimysial fibrosis.

Conclusions: The predominat features of SSc-related myopathy are fibrosis, microangiopathy and humoral immunity. However, it is difficult to identify specific histopathological hallmarks of muscle involvement in SSc, since they could be present also in other (IIM/NIM) myopathies.

Disclosure of Interest: None declared

DOI: 10.1136/annrheumdis-2017-eular.4070

\section{OP0083 MOLECULAR TARGETED IMAGING BIOMARKERS FOR PERSONALIZED MEDICINE STRATEGIES IN SYSTEMIC SCLEROSIS-RELATED INTERSTITIAL LUNG DISEASE}

J. Schniering ${ }^{1}$, M. Benešová ${ }^{2}$, M. Brunner ${ }^{1}$, C. Feghali-Bostwick ${ }^{3}$, R. Schibli ${ }^{2}$, O. Distler ${ }^{1}$, C. Müller ${ }^{2}$, B. Maurer ${ }^{1} .{ }^{1}$ Department of Rheumatology, University Hospital Zurich, Zurich; ${ }^{2}$ Center for Radiopharmaceutical Sciences, Paul Scherrer Insitute, Villigen-PSI, Switzerland; ${ }^{3}$ Division of Rheumatology \& Immunology, Medical University of South Carolina, Charleston, SC, United States

Background: Interstitial lung disease (ILD) is a life-threatening complication in systemic sclerosis (SSc). Substantial research progress has identified distinct genomic and molecular subtypes in SSc-ILD and brought molecular targeted therapies within reach. However, personalized medicine approaches are still lacking since clinically applicable tools for individualized patient stratification are not yet available.

Objectives: To assess the possibility of imaging molecular targets as a biomarker for stage-dependent assessment of ILD in the mouse model of bleomycin-induced lung fibrosis.

Methods: Expression of integrin $\alpha_{\mathrm{v}} \beta_{3}$ and folate receptor $\beta$ (FR- $\beta$ ) was analyzed in lung tissues from patients with idiopathic pulmonary fibrosis (IPF), SSc-ILD, and healthy controls as well as from bleomycin treated mice and saline treated controls using immunohistochemistry and GPCR $(n=5-11)$. SPECT (Single Photon Emission Computed Tomography) or PET (Positron Emission Tomography) was performed at days 3,7 , and 14 after bleomycin instillation using the integrin $\alpha_{v} \beta_{3}-$ specific ${ }^{177} \mathrm{Lu}-\mathrm{c}(\mathrm{RGDFK})$-ligand and the FR- $\beta$-specific ${ }^{18} \mathrm{~F}$-Azafol. Additionally, ${ }^{18}$ F-FDG-PET and high resolution CT (HRCT) scans were performed. The specific lung uptake of the radiotracers over time was assessed by ex vivo SPECT or PET/CT scans and biodistribution studies.

Results: Expression of FR- $\beta$ was significantly increased at the mRNA and protein level of SSc-ILD patients as compared to healthy subjects $(p<0.05)$, whereas only its gene expression was upregulated in IPF patients $(p<0.01)$. Integrin $\alpha_{\nu} \beta_{3}$ was increased at the protein level of both SSc-ILD and IPF patients $(p<0.05)$, while its mRNA expression was not significantly altered.

Similarly, in lungs of bleomycin treated mice, but not of controls, FR- $\beta$ expression was increased time-dependently at the mRNA and protein level with higher expression at day 3 and day 7 , the inflammatory stages of bleomycin-induced lung fibrosis $(\mathbf{p}<0.05)$. In contrast, expression of integrin $\alpha_{4} \beta_{3}$ was upregulated at day 7 and day 14 at the protein, but not at the mRNA level in bleomycin treated mice, and thus not only in the inflammatory but also in the fibrotic stages $(p<0.05)$. ${ }^{18} \mathrm{~F}-\mathrm{FDG}$-PET and HRCT detected time-dependent changes of metabolic activity and ILD morphology in bleomycin treated mice. However, compared with these routinely employed unselective imaging techniques, molecular targeted imaging of integrin $\alpha_{v} \beta_{3}$ and FR- $\beta$ successfully visualized ILD and discriminated lung inflammation and/or fibrosis in a time-dependent manner and in correspondence with the expression changes at the tissue level. The specific lung uptake of ${ }^{177} \mathrm{Lu}-\mathrm{c}(\mathrm{RGDfK})$-ligand and ${ }^{18} \mathrm{~F}$-Azafol as compared with the unspecific uptake of ${ }^{18} \mathrm{~F}-\mathrm{FDG}$ in diseased lungs over time was demonstrated by biodistribution studies and ex vivo SPECT or PET/CT scans.

Conclusions: Our data suggest that stage-dependent visualization of ILD with radiotracers that target key markers of lung inflammation and/or fibrosis shows promise for clinical application. As opposed to unselective imaging techniques such as ${ }^{18} \mathrm{~F}$-FDG-PET and HRCT, the introduction of specific imaging biomarkers for individualized management of SSc-ILD patients could represent the first step towards precision medicine.

Disclosure of Interest: J. Schniering Grant/research support from: Swiss National Science Foundation (S-85605-02-01), M. Benešová Grant/research support from: Swiss National Science Foundation (S-85605-02-01), M. Brunner: None declared, C. Feghali-Bostwick: None declared, R. Schibli Grant/research support from: Merck \& Cie, O. Distler Grant/research support from: Actelion, Bayer, Boehringer Ingelheim, Pfizer, Sanofi; Patent licensed: mir-29 for the treatment of systemic sclerosis, Consultant for: 4 D Science, Actelion, Active Biotec, Bayer, Biogenldec, BMS, Boehringer Ingelheim, ChemomAb, EpiPharm, espeRare foundation, Genentech/Roche, GSK, Inventiva, Lilly, medac, Mepha, Medlmmune, Mitsubishi Tanabe Pharma, Pharmacyclics, Pfizer, Sanofi, Serodapharm, Sinoxa, Speakers bureau: AbbVie, iQone Healthcare, Mepha, C. Müller Grant/research support from: Merck \& Cie, B. Maurer Grant/research support from: AbbVie, Protagen, EMDO, Novartis, Pfizer, Roche, Actelion; Patent licensed: mir-29 for the treatment of systemic sclerosis

DOI: 10.1136/annrheumdis-2017-eular.1871

\section{OP0084 RESCUE FROM THE FAILING HEART IN SYSTEMIC SCLEROSIS, A NOVEL INSIGHT: TARGETING TGF- $\beta$ /FRA2-DEPENDENT AUTOPHAGY}

M. Stellato ${ }^{1}$, M. Rudnik ${ }^{1}$, F. Renoux ${ }^{1}$, E. Pachera ${ }^{1}$, D. Kayalar ${ }^{1}$, K. Sotlar ${ }^{2}$, K. Klingel $^{3}$, J. Henes ${ }^{4}$, P. Blyszczuk ${ }^{5}$, O. Distler ${ }^{1}$, G. Kania ${ }^{1}$. ${ }^{1}$ Department of Rheumatology, University Hospital, Zurich, Switzerland; ${ }^{2}$ Institute of Pathology Ludwig Maximilians University, Munich; ${ }^{3}$ Department of Molecular Pathology; ${ }^{4}$ Division of Rheumatology, University Hospital, Tubingen, Germany;

${ }^{5}$ Department of Clinical Immunology, Jagiellonian University Medical College, Krakow, Poland

Background: The majority of Systemic Sclerosis (SSc) patients have subclinical primary cardiac involvement, which resembles the inflammatory dilated cardiomyopathy (iDCM) with inflammation and fibrosis. Firstly, cellular progenitors of pathological myofibroblasts remain undescribed.

Secondly, autophagy may favor fibrosis through enhanced differentiation of fibroblasts in myofibroblasts

Objectives: To unravel the role of Fos-related antigen 2 (Fra2)/autophagy crosstalk in TGF- $\beta$-driven myocardial fibrosis in SSc

Methods: Genetically modified mice overexpressing Fra2 were used. Endomyocardial biopsies (EMBs) from SSc/iDCM patients and hearts from Fra2 tg and control mice were analysed by immunohistochemistry $(\mathrm{IHC})$ and immunofluorescence (IF). Murine myocardial gp38+ podoplanin $^{+}$) stromal cells were sorted and stimulated in vitro with TGF- $\beta$. The cellular phenotype was assessed by GPCR, IF, stress fiber staining, SIRCOL and contraction assay on sorted cells. The antisense oligonucleotide GapmeR was used to knock-down Fra2

Results: The myocardium of SSc/iDCM patients $(n=10)$ showed the typical phenotype of iDCM: increased fibrotic area with extended collagen deposition and enlarged number of pathological myofibroblasts, expressing Fra2 and, interestingly, the autophagy markers LC3B and Atg5. Similarly, Fra2 tg mice showed higher expression of profibrotic markers: $\alpha$ SMA, vimentin and collagen I compared to control mice $(n=5)$, as well as the expression of the autophagy markers LC3B and Beclin in fibrotic regions.

Among cardiac stromal cells (Ter119-CD $45^{-}$CD $311^{-S c a} 1^{+}$CD $\left.29^{+}\right)$the frequency of gp $38^{+}$cells was significantly higher in Fra2 myocardium compared to control mice. The majority of gp $38^{+}$cells co-expressed $\alpha \mathrm{SMA}$, vimentin and collagen I together with autophagy markers ( $\mathrm{LC} 3 \mathrm{~B}$ and Beclin, $\mathrm{n}=3$ ). Following in vitro stimulation with TGF- $\beta$, gp $38^{+}$cells entered fibroblast-to-myofibroblast transition characterized by increased mRNA and protein levels of aSMA, collagen I, fibronectin ( $n=3-6), \alpha S M A$-fiber and stress-fiber formation $(n=3)$, increased cell proliferation $(n=4 ; p=0.04)$ and contraction capability $(n=2 ; p<0.05)$ and enhanced collagen secretion $(n=3 ; p=0.04)$. Importantly, Fra2 tg gp $38^{+}$cells showed the presence of $\alpha$ SMA and stress fibers even without TGF- $\beta$ stimulation as well as an increased contraction capability compared to control cells.

TGF- $\beta$ stimulation of control gp $38^{+}$cells induced the expression of LC3B, Beclin and Atg 5 at mRNA and protein level $(n=3-5)$. In contrast, TGF- $\beta$ inhibition caused the downregulation of these markers $(n=3)$.

In addition, Fra2 silencing resulted in a decreased differentiation capability of 